\title{
Physician's Satisfaction from Laboratory Services in Maternity and Children Hospital in Makkah
}

\author{
Rana G. Zaini ${ }^{1}$ and Rania G. Zaini ${ }^{*}$
}

${ }^{1}$ Department of Medical Laboratories, College of Applied Medical Sciences, Taif University, Taif, Kingdom of Saudi Arabia

${ }^{2}$ Medical Education Department, Faculty of Medicine, Umm AlQura University, Makkah, Kingdom of Saudi Arabia

\begin{abstract}
Background: Physicians are primary customers of laboratory services and their perception of the provided services is considered an important measure of quality assurance. This study investigates the physicians' satisfaction of laboratories' services, in Maternity and Children Hospital in Makkah, to identify strength and possible limitations, which might inform the development plans for more efficient services.

Methods: The study measured satisfaction of laboratories' primary customer (physicians) of provided services in fourfold: quality of reports, communication, management, and TAT. Paper-based survey of 5-Likert Scale, ranged from Strongly Disagree (1) to Strongly Agree (5) was developed based on the CAP survey and related published studies. The questionnaire was piloted and then demonstrated between May and June 2014.

Results: Sixty-three physicians complete the survey. The overall satisfaction of the provided services were limited with mean score 64/120. Respondents were not pleased with the majority of items with mean ranged between 3.4 (Unsure) to 2.3 (Disagree). Physicians were most satisfied with courtesy of laboratory personnel $($ mean $=3.4)$, accuracy of laboratory results $($ mean $=3.2)$ and staff support to research projects $($ mean $=3.1)$. However, the lowest satisfaction rete was reported with the test turnaround times (TAT) for state, and routine tests for inpatient and outpatient.

Conclusion: This study presented a number of negative observations of laboratory services at the Maternity and Children Hospital in Makkah, which mainly related to administration, communication, quality and delay in TAT. The efficiency and optimization of laboratory service need to be readdressed by the laboratory administration. Effective extra laboratory communication channels needed to be established to improve interaction between laboratory and physicians. Point-of-care testing (POCT) is one of the promising implementing to improve TAT since, no time required for sample's preparation and transportation.
\end{abstract}

\section{Introduction}

Hospital laboratories are essential component and one of the most important departments at any healthcare services where medical tests and investigations done in order to generate reliable and accurate information regarding patient's health [1]. Laboratory reports usually are the bases of medical decisions and possible management plans that considered by physicians. Therefore, laboratory results must be of the highest quality and reliability to insure that the course of action taken by physicians will almost lead to the best possible outcome for the patient $[1,2]$.

Customers' satisfactions of the provided services, such as in the healthcare institutes, are considered one of the essential key performance indictor of quality $[3,4]$. The World Health Organization (WHO) indicates that evaluations of client satisfaction might address various aspects of the provided services: reliability and consistence of the services, the responsiveness of services, and the willingness of providers to meet client's expectations and needs [5]. Thus, the efficiency of laboratories could be measured from different perspective. Physicians are the primary customers of laboratory services and their satisfaction of provided services is an important quality measure in most quality assurances frameworks $[4,6]$. According to the American Pathologist (CAP) and the Joint Commission on Accreditation for Healthcare Organization's laboratory checklist, physicians are considered the primary customer of laboratories' services and measuring their satisfaction is an essential concept [6]. In the United States, most clinical laboratories are required to assess satisfaction of customers in order to validate and maintain accreditation [6,7]. These surveys carried out periodical to characterize limitations, progress, and changes in laboratory services performances.

Many aspects of the laboratory services could be investigated from the perspective of physicians including, quality/reliability of test results, staff courtesy, accessibility of pathologist, accessibility of laboratory manager, phlebotomy services, test menu adequacy, accessibility of laboratory staff, courier services, routine test turnaround time (TAT), laboratory management responsiveness, inpatient stat test TAT, critical value notification, clinical report format, outpatient stat test TAT, and esoteric TAT [6]. In addition, one of the most obvious indications of laboratory service, which often used as a crucial performance indicator of laboratory performance, is turnaround time (TAT) [8]. TAT is the time to return a test result $[8,9]$. Assessment of TAT allows laboratory directors to understand whether local performance is adequate or need to be improved, and how it is compared with published norms. Delays in reporting laboratory results can lead to delay in the management, diagnosis, treatment and release of patients $[9,10]$.

Various studies investigated the satisfaction of the primary

"Corresponding Author: Dr. Rania G. Zaini, Department of Medical Laboratories, College of Applied Medical Sciences, Taif University, Taif, Kingdom of Saudi Arabia; E-mail: ranazaini2@tu.edu.sa

Citation: Zaini RG, Zaini RG (2015) Physician's Satisfaction from Laboratory Services in Maternity and Children Hospital in Makkah. Int J Lab Med Res 1: 101 doi: http://dx.doi.org/10.15344/2455-4006/2015/101

Copyright: () 2015 Zaini et al. This is an open-access article distributed under the terms of the Creative Commons Attribution License, which permits unrestricted use, distribution, and reproduction in any medium, provided the original author and source are credited. 
Citation: Zaini RG, Zaini RG (2015) Physician's Satisfaction from Laboratory Services in Maternity and Children Hospital in Makkah. Int J Lab Med Res 1: 101. doi: http://dx.doi.org/10.15344/2455-4006/2015/101

Page 2 of 4

healthcare providers (physicians) of laboratory services to identify possible limitations for future development $[6,11,12]$. For example, in 2002, Tegbaru and colleagues, assessed 28 hospital and six regional laboratories in Ethiopia and reported number of problems mainly related to the limited infrastructure such as, lack of properly designed laboratory rooms, lack of water and electricity access, shortage of equipment and supplies, and absence of effective maintenance and spare parts. Also poor supervision and follow up were reported [12]. Within the same study the lowest rate of satisfaction were found for critical value notification [12]. On the other hand, another studies showed that the critical value notification, quality of laboratory results and staff courtesy were reported as the highest level of satisfaction, while the least satisfaction was seen in the TAT $[13,14]$.

In 2001, a study evaluated the satisfaction of the physicians at the Emergency Department (ED) and reported that physicians are not satisfied with laboratory services, since the laboratory TAT caused delayed ED treatment and increased length of stay in ED [10]. This concurs with Hawkins, (2007) findings that laboratory testing performed with long TAT affected patient release [8]. Many laboratories have had difficulties improving their TATs and no clear reasons for prolonged TATs are defined $[9,15]$. More recent study done in six hospitals ( 3 public and 3 private) in Aden and showed that the lowest satisfaction score was reported from TAT, however, physicians in private institutes showed a higher satisfaction level [4]. Mostly, staff shortages found to be associated with long TAT as a result of delay in the test ordering and collection [15]. In contrast, a study performed in 653 institutions, participating in the College of American Pathologists Q-Probes program, for inpatients early morning routine clinical laboratory tests and found little evidence that longer routine test TAT affects patient length of stay [16]. On the other hand, faster TAT does not necessarily improving patient outcome as reported at Howanitz's study [17]

This study aimed to investigate physicians' satisfaction of hospital clinical laboratories' services. The study might be indicators of possible limitations and the bases of quality improvement, which would lead to efficient healthcare services. This study also, measures the satisfaction of laboratories' primary customer (physicians) of provided services in fourfold: quality of reports, communication, management, and TAT.

\section{Method}

Physician satisfaction paper-based survey of 5 -Likert Scale, $(1=$ Strongly Disagree, $2=$ Disagree, $3=$ Unsure, $4=$ Agree, and 5= Strongly Agree), was designed based on the CAP survey and other published similar studies. The questionnaire consisted of 24 questions, evaluating different aspects of health care services including; the accuracy of report, the effectiveness of laboratory team's communications, the efficiency of laboratory management, and the TAT. The questionnaire was piloted on three physicians and academics of medical schools, and modifications were applied. Data was analyzed with SPSS version 19.

Study population was physicians of the Maternity and Children Hospital in Makkah. The study proposal was approved by the Research Ethical Committee of the Maternity and Children Hospital in Makkah and conducted between the period of May and June 2014. The survey was disseminated to all hospital's departments and physicians were invited to participate. In addition, physician were approached individually and asked to complete the survey, which will lead to better laboratory services and quality.

\section{Results}

The study was completed by Sixty-three physicians. The majority were Saudi $67 \%(\mathrm{~N}=42)$, male $57 \%(\mathrm{~N}=36)$, and with less than sixyear experience $66 \%(\mathrm{~N}=37)$. About two third of the respondents were Pediatricians $71 \%(\mathrm{~N}=45)$, and $29 \%$ were Obstetricians $(\mathrm{N}=18)$. Also, most respondents were residents or specialist, $46 \%$ and $23 \%$, respectively. The statistical reliability of the survey item was good as measured with Cronbach's Alpha (0.7) [18].

The study showed respondents relative dissatisfaction with total mean score of 64/120. The overall mean rating of satisfaction by physicians in the Maternity and Children Hospital in Makkah ranged between $2.3-3.4$ out of 5 . According to the study results, respondents to physician satisfaction survey indicated the greatest satisfaction rate related to courtesy of laboratory personnel $($ mean $=3.4)$, accuracy of laboratory results (mean $=3.2$ ), and staff support to research projects $($ mean $=3.1)$. Followed by the adequacy of laboratory point of care testing support $($ mean $=3.0)$ and the format of clinical reports (mean= 3.0) (Table 1).

Also the analysis showed negative perception for the accessibility of mangers (mean $=2.6$ ), staff availability $(2.8)$, and handling telephone enquires (mean $=2.5$ and 2.3) (Table 1). Moreover, respondents expressed their dissatisfaction of the laboratories essential instructions and guides, such as the notification of changes, the manual and protocol guide, and the references value booklets (Table 1). A major area of respondents' negative perception of laboratory services was the TAT for state tests, and routine test for inpatient and outpatient (ranged 2.5-2.6) (Table 1).

\section{Discussion}

The objective of the survey was to explore strengths and limitations of laboratories' services in order to develop appropriate action plans that concur with the institute mission and vision. The questionnaire was developed based on previous studies and the CAP survey. It involved several statements covering different sections and details of laboratory services, which were considered important to physicians as primary customers for the laboratory hospital.

As in a number of studies found that the reliability and accuracy of the laboratory results were most important statements for physician as laboratory's customers [6]. This study reported respondents' general satisfaction of the accuracy and reliability of study results. This finding concurs with other studies $[13,19]$. It seems that the reliability of the test results is very adequate in most well established hospitals.

Many aspects related to laboratory staff communication, such as staff availability, handling telephone enquiries, and answering enquires regarding missing test results, were not perceived satisfactory by respondents. In many institutes this was not a considered one of the limitation [6]. Establishing a clear communication means is essential aspect could be considered by the laboratory administration. Also, finding indicated the need for more instruction and guidelines, such as preparation of patients for laboratory tests, and the collection and handling of samples since the respondents to the survey expressed their dissatisfaction toward the laboratories instructions and guides, such as the notification of changes may affects samples collection and generating patients results, the manual and protocol guide and the references value booklets. The satisfaction with management of laboratory showed a very low satisfaction level, there is a major need of dynamic communication between laboratory and physicians especially with unclear, mistake and missing results. 
Citation: Zaini RG, Zaini RG (2015) Physician's Satisfaction from Laboratory Services in Maternity and Children Hospital in Makkah. Int J Lab Med Res 1: 101. doi: http://dx.doi.org/10.15344/2455-4006/2015/101

\begin{tabular}{|c|c|c|c|}
\hline & Statements & Mean & S.D \\
\hline 1. & Laboratory results are accurate & 3.2222 & .97459 \\
\hline 2. & Laboratory services are efficient & 2.7619 & 1.20100 \\
\hline 3. & $\begin{array}{l}\text { Laboratory notification of the changes in } \\
\text { services is adequate }\end{array}$ & 2.5397 & 1.05991 \\
\hline 4. & $\begin{array}{l}\text { Laboratory manuals and protocols for } \\
\text { investigation time are adequate }\end{array}$ & 2.2540 & 1.01550 \\
\hline 5. & Reference value booklet is fit for use & 2.6984 & 1.17274 \\
\hline 6. & Laboratories' management is accessible & 2.6667 & 1.12163 \\
\hline 7. & Laboratory staff are available & 2.8413 & 1.11016 \\
\hline 8. & $\begin{array}{l}\text { Laboratories usual promptly answered } \\
\text { telephones calls }\end{array}$ & 2.3016 & 1.22652 \\
\hline 9. & $\begin{array}{l}\text { Laboratories answered efficiently most of } \\
\text { our telephones' enquires }\end{array}$ & 2.5238 & 1.13389 \\
\hline 10. & $\begin{array}{l}\text { Laboratory point of care testing support } \\
\text { is adequate }\end{array}$ & 3.0000 & .98374 \\
\hline 11. & $\begin{array}{l}\text { Turnaround time (TAT) is adequate for } \\
\text { stat tests }\end{array}$ & 2.5556 & 1.11843 \\
\hline 12. & $\begin{array}{l}\text { Turnaround time (TAT) is adequate for } \\
\text { routine tests for in-patients }\end{array}$ & 2.6190 & 1.06904 \\
\hline 13. & $\begin{array}{l}\text { Turnaround time (TAT) is adequate for } \\
\text { routine tests for outpatients }\end{array}$ & 2.6667 & 1.16398 \\
\hline 14. & Abnormal results notification is adequate & 2.5714 & 1.22756 \\
\hline 15. & $\begin{array}{l}\text { Service attitude of the laboratory } \\
\text { personnel is good }\end{array}$ & 3.4286 & 1.11752 \\
\hline 16. & $\begin{array}{l}\text { Laboratory has a positive attitude toward } \\
\text { our research projects }\end{array}$ & 3.1429 & 1.07549 \\
\hline 17. & $\begin{array}{l}\text { The format of laboratories' clinical report } \\
\text { are convenient }\end{array}$ & 3.1270 & 1.00791 \\
\hline 18. & $\begin{array}{l}\text { We are satisfied with the laboratory } \\
\text { information system when requesting } \\
\text { laboratory tests }\end{array}$ & 2.7302 & 1.22087 \\
\hline 19. & $\begin{array}{l}\text { We are satisfied with the laboratory } \\
\text { information system when reviewing } \\
\text { laboratory results in electrical patient } \\
\text { records }\end{array}$ & 2.6508 & 1.20695 \\
\hline 20. & $\begin{array}{l}\text { We do not need additional instructions } \\
\text { on the preparation of patients for } \\
\text { laboratory tests }\end{array}$ & 2.5238 & 1.13389 \\
\hline 21. & $\begin{array}{l}\text { We do not need additional instructions } \\
\text { on the collection and handling of } \\
\text { samples }^{*}\end{array}$ & 2.3810 & 1.11339 \\
\hline 22. & $\begin{array}{l}\text { We do not need additional instructions as } \\
\text { consultations by laboratory physicians }{ }^{*}\end{array}$ & 2.4444 & 1.17470 \\
\hline 23. & $\begin{array}{l}\text { We rarely need to contact the laboratory } \\
\text { because of missing test results }\end{array}$ & 2.0159 & 1.27624 \\
\hline 24. & $\begin{array}{l}\text { We rarely need to contact the laboratory } \\
\text { because of erroneous test results* }\end{array}$ & 2.26 & 1.24701 \\
\hline $\begin{array}{l}\text { Total } \\
\text { Mean }\end{array}$ & & 63.92 & \\
\hline
\end{tabular}

Table 1: Results of the survey

* Reversed value

Within this study, most physicians have encountered their dissatisfaction from abnormal results notification. This concurs Gondar University Hospital survey in northwest Ethiopia [20]. Thus, there is a call to improve the abnormal results notification systems and information system to request laboratory results or review results in electronic patient records.

In addition, TAT is one of the most noticeable indications of laboratory service and is often used as a key performance indicator of laboratory performance, however, it is common for laboratories to hear from dissatisfied users that their test TATs generally are not fast enough [20]. In this study, physicians were not pleased with TAT for both state and routine tests for inpatient and outpatient: thus concurs with number of studies in the United States of America [6,10], and the Eastern Mediterranean Region [4, 9, 21]. Most common reasons for TAT delay were found to be shortage of highly trained personnel and machine breakdown followed by problems in machine maintenance $[15,21]$.

One possible solution that may decrease TAT is point-of-care testing (POCT), which is a laboratory investigation or analyses performed by non-laboratory healthcare professionals. TAT can be improved as delays are no longer caused by sample transport and preparation also the results are available more quickly [22]. In the United States, Lewandrowski and colleagues evaluated the physician satisfaction with TAT at emergency department of the Massachusetts General Hospital, Boston, before and after implementation of a POCT laboratory and found that the TAT declined with an average of more than $85 \%$ after the institution of POCT [23].

This study had some limitations. It is the first study carried out in Maternity and Children Hospital in Makkah based on doctors' satisfaction for the services provided by the hospital's laboratories. It was conducted in one hospital and one city, thus the results could not be generalized. Also the study considered one laboratory services customer "Physicians": other studies need to investigate the perception of other laboratory customers including, nurse and patients. Poor respondents rate of hospital physicians was also reported.

\section{Conclusions}

The study results presented that the physicians in the Maternity and Children Hospital in Makkah are not satisfied with the efficiency of services provided by the laboratory. Major concern was raised on aspect related to administration, communication, quality and delay in TAT. The efficiency and optimization of laboratory service need to be readdressed by the laboratory administration. Efficient medical laboratory services required more than skillful laboratory team. Laboratory specialists and team must be competent in all aspects; knowledgeable, skillful, professional, communicator, scholar, and leader. Within the current promising working environment and leadership of the Maternity and Children Hospital in Makkah, which considered the holistic approach of developing provided services, there are many aspects to be developed, the first and crucial one to start is culture awareness of the laboratory services, plans and goals, and then build and maintain a clear communication schema between laboratory personnel and targeted customers. Although it appears that one issue may relate to the other, the interaction between the laboratory and the physicians is quite complex. In addition there is a need to engage all related customers: nurses, patients, physicians, and administration in the process of development.

\section{Author Contributions}

Rana G. Zaini, contributed in the study design, ethical approval, data collection and encoding, interpretation of data, and drafting the manuscript.

Rania G. Zaini, contributed in the study Design, analysis and interpretation of data, and reviewing the manuscript.

\section{Competing Interests}

The authors have declared that no competing interests exist. 
Citation: Zaini RG, Zaini RG (2015) Physician's Satisfaction from Laboratory Services in Maternity and Children Hospital in Makkah. Int J Lab Med Res 1: 101. doi: http://dx.doi.org/10.15344/2455-4006/2015/101

\section{References}

1. Hassemer DJ (2003) Wisconsin State Laboratory of Hygiene's role in clinical laboratory improvement. WMJ 102: 56-59.

2. Teklemariam Z, Mekonnen A, Kedir H, Kabew G (2013) Clients and clinician satisfaction with laboratory services at selected government hospitals in eastern Ethiopia. BMC Res Notes 6: 15

3. Kairys J, Zebiene E, Sapoka V, Zokas I (2008) Satisfaction with organizational aspects of health care provision among Lithuanian physicians. Cent Eur J Public Health 16: 29-33.

4. Adulkader NM, Triana BE (2013) Physician satisfaction with hospital clinical laboratory services in Aden Governorate, Yemen, 2009. East Mediterr Health J 19: 555-560.

5. Workbook 6. Client Satisfaction Evaluations. Geneva, World Health Organization, 2000

6. Bruce JA, Bekeris LG, Raouf E. Nakhleh, Walsh MK, et al. (2009) Physician Satisfaction With Clinical Laboratory Services: A College of American Pathologists Q-Probes Study of 138 Institutions. Arch Pathol Lab Med133: 38-43.

7. Nakhleh RE, Souers R, Ruby SG (2008) Physician satisfaction with surgical pathology reports: a 2-year College of American Pathologists Q-Tracks Study. Arch Pathol Lab Med 132: 1719-1722.

8. Hawkins RC (2007) Laboratory turnaround time. Clin Biochem Rev 28 179-194.

9. Elhoseeny TA, Mohammad EK (2013) Quality of the clinical laboratory department in a specialized hospital in Alexandria, Egypt. East Mediterr Health J 19: 81-87.

10. Steindel SJ, Howanitz PJ (2001) Physician Satisfaction and Emergency Department Laboratory Test Turnaround Time. Arch Pathol Lab Med 125 863-871.

11. Oja PI, Kouri TT, Pakarinen AJ (2006) From customer satisfaction survey to corrective actions in laboratory services in a university hospital. Int J Qual Health Care 18: 422-428.

12. Tegbaru B, Meless H, Kassu A, Desalegn T, Gezahegn N, et al. (2004) Laboratory services in hospitals and regional laboratories in Ethiopa. Ethiop J Health De 18: 43-47.

13. Miller KA, Dale JC (1999) Physician Satisfaction with Clinical Laboratory Service, 99-03: Q-Probes. Northfield, III: College of American Pathologists.

14. Howanitz PJ (2002) Physician Satisfaction With Clinical Laboratory Services: Q-Probes Data Analysis and Critique. Northfield, III: College of American Pa- thologists.

15. Steindel SJ, Novis DA (1999) Using outlier events to monitor test turnaround time. Arch Pathol Lab Med 123: 607-614.

16. Steindel SJ, Jones BA, Howanitz PJ (1996) Timeliness of automated routine laboratory tests: a College of American Pathologists Q-Probes study of 653 institutions. Clin Chim Acta 251: 25-40.

17. Howanitz PJ (2005) Errors in laboratory medicine: practical lessons to improve patient safety. Arch Pathol Lab Med 129: 1252-1261.

18. Tavakol M, Dennick R (2011) Making sense of Cronbach's alpha. Int J Med Educ 2: 53-55

19. Koh YR, Kim SY, Kim IS, Chang CL, Lee EY, et al. (2014) Customer satisfaction survey with clinical laboratory and phlebotomy services at a tertiary care unit level. Ann Lab Med 34: 380-385.

20. Addis Z, Birhan W, Derseh D, Sahle B, Gizaw N (2013) Physicians' and nurses' satisfaction with the clinical laboratory service of Gondar University Hospital, northwest Ethiopia. Am J Clin Pathol 140: 324-328.

21. Bilwani F, Siddiqui I, Vaqar S (2003) Determination of delay in burn around time (TAT) of stat tests and its causes: an AKUH experience. J Pak Med Assoc 53: 65-67.

22. Luppa PB, Müller C, Schlichtiger A, Schlebusch H (2011) Point-of-care testing (POCT): Current techniques and future perspectives. TrAC Trends Analyt Chem 30: 887-898.
23. Lewandrowski EL, Corboy D, Lewandrowski K, Sinclair J, McDermot S, et al. (2003) Implementation of a Point-of-Care Satellite Laboratory in the Emergency Department of an Academic Medical Center. Impact on test turnaround time and patient emergency department length of stay. Arch Pathol Lab Med 127: 456-460. 